\title{
Time-of-Flight Measurement of Resonant Molecular Formation in Muon Catalyzed $d t$ Fusion
}

G.M. Marshall ${ }^{1}$, A. Adamczak ${ }^{2}$, J.M. Bailey ${ }^{3}$, G.A. Beer ${ }^{4}$, J.L. Beveridge ${ }^{1}$, J.L. Douglas ${ }^{4}$, M.P. Faifman ${ }^{5}$, M.C. Fujiwara ${ }^{6}$, T.M. Huber ${ }^{7}$

R. JaCOT-Guillarmod ${ }^{8}$, P. KammeL ${ }^{9}$, N.P. Kherani ${ }^{10}$, S.K. Kim ${ }^{11}$

P.E. Knowles ${ }^{4}$, A.R. Kunselman ${ }^{12}$, M. Maier ${ }^{4}$, V.E. Markushin ${ }^{5}$, C.J. Martoff ${ }^{13}$, G.R. Mason ${ }^{4}$, V.S. Melezhik ${ }^{14}$, F. Mulhauser ${ }^{1, *}$, A. Olin ${ }^{4}$, C. Petitjean ${ }^{15}$, C. Piller ${ }^{8}$, T.A. Porcelli ${ }^{4}$, C.P. Steffens ${ }^{7}$, And J. Zmeskal 16

${ }^{1}$ TRIUMF, Vancouver, BC, V6T $2 A 3$ Canada

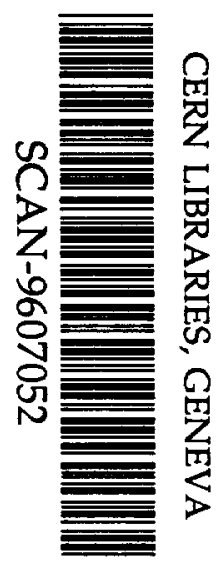

${ }^{2}$ INP, 31-342 Krakow, Poland

${ }^{3}$ Chester Technology, Chester, UK

${ }^{4}$ University of Victoria, Victoria, $B C, V 8 W 2 Y 2$ Canada

${ }^{5}$ RRC Kurchatov Institute, Moscow 123182, Russia

${ }^{6}$ University of British Columbia, Vancouver, BC, V6T 2A6 Canada

${ }^{7}$ Gustavus Adolphus College, St. Peter, MN 56082, USA

${ }^{8}$ University of Fribourg, CH-1700 Fribourg, Switzerland

${ }^{9} L B L$, Berkeley, CA 94720, USA

${ }^{10}$ Ontario Hydro, Toronto, ON, M8Z $5 S 4$ Canada

${ }^{11}$ Jeonbuk National University, Jeonju 560-756, Korea

${ }^{12}$ University of Wyoming, Laramie, WY 82071, USA

${ }^{13}$ Temple University, Philadelphia, PA 19122, USA

${ }^{14}$ JINR, Dubna 141980, Russia

${ }^{15}$ Paul Scherrer Institute, CH-5232 Villigen, Switzerland

${ }^{16}$ IMEP, Austrian Academy of Sciences, A-1090 Wien, Austria

*Present address: University of Fribourg, CH-1700 Fribourg, Switzerland 
Preliminary results are reported for an experiment at TRIUMF where a time-offlight technique was tested for measuring the energy dependence of the rate for muon catalyzed $d t$ fusion. Muonic tritium atoms were created following transfer of negative muons from muonic protium in a layer of solid hydrogen (protium) containing a small fraction of tritium. The atoms escaped from the solid layer via the Ramsauer-Townsend mechanism, traversed a drift region of $18 \mathrm{~mm}$, and then struck an adjacent layer of deuterium, where the muonic atom could form a molecular system.

The time of detection of a fusion product (neutron or alpha) following muon arrival is dependent upon the energy of the muonic tritium atom as it traverses the drift region. By comparison of the time distribution of fusion events with a prediction based on the theoretical energy dependence of the rate, the strength of resonant formation can in principle be determined. The results extracted so far are discussed and the limitations of the method are examined.

Keywords: muon catalyzed fusion, muonic hydrogen, muonic atoms, muonic molecules.

\section{Introduction}

The use of solid hydrogen isotopes and isotope mixtures as the target medium for experiments on muonic hydrogen has developed substantially in the past few years. One of the advantages is that targets can be designed specifically for a given measurement by creating layers of different isotopic composition, so that the interactions of the muonic system with different isotopes can in some cases be separated in space or time. While interesting and surprising results have been obtained with pure solid deuterium[1,2], many of the recent experiments $[3,4,5]$ and proposals $[6,7]$ take advantage of energetic muonic deuterium $(\mu d)$ or tritium $(\mu t)$ atoms which result when small amounts of deuterium or tritium are mixed with protium. Recently the experiments have been simulated via Monte Carlo methods $[8,9,10]$ using as input the calculated values for processes such as scattering, transfer, and molecular formation.

Resonant formation speeds the process of combining, for example, tritons and deuterons into $d \mu t$ muonic molecular ions, from which fusion can proceed rapidly. In the resonance mechanism, the kinetic energy of a muonic tritium atom is absorbed in the excited state of the muonic molecular complex, as in

$$
\mu t+\mathrm{DX} \rightarrow\left[(\mathrm{d} \mu \mathrm{t})^{+} \mathrm{xee}\right]^{*},
$$

where $x(\mathrm{X})$ represents $p, d$, or $t(\mathrm{H}, \mathrm{D}$, or $\mathrm{T})$. These reactions exhibit the highest resonant formation rates predicted in any muon catalyzed fusion $(\mu \mathrm{CF})$ cycle, at a laboratory kinetic energy for $\mu t$ above $0.4 \mathrm{eV}$. Figure 1 shows the effective rates calculated for $\mu t+\mathrm{D}_{2}$ using the method of Faifman and Ponomarev[11] extended to a target temperature of $3 \mathrm{~K}$. More recently, the inclusion of quadrupolar terms has reduced the calculated effective rates by typically $10-15 \%[12]$. Similar results have been obtained independently for the $F=0$ hyperfine state of $\mu t[13]$. Because the main resonances are far above thermal energy at ambient or practical laboratory temperatures, and because a substantial amount of tritium is required, it is a significant technical challenge to obtain abundant muonic atoms in a deuterium-tritium target in this energy range by simply elevating the temperature 


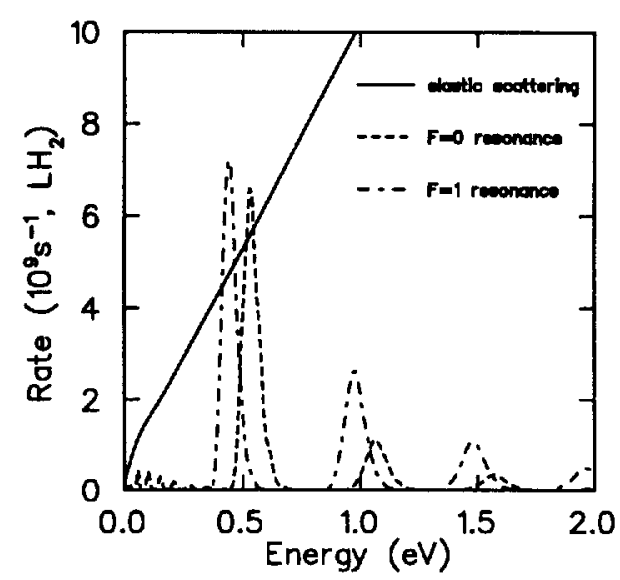

Figure 1: Predicted resonance structure of $\mu t+\mathrm{D}_{2}$ at $3 \mathrm{~K}$ and density of liquid hydrogen[11], compared with rate of energy loss, for two hyperfine states of $\mu t$.

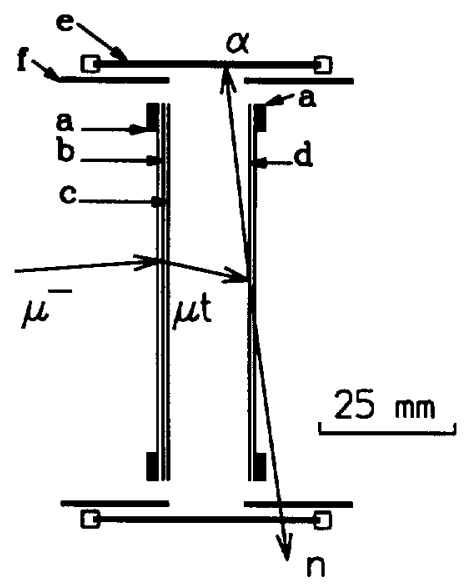

Figure 2: Diagram of the target: (a) gold foil and support, (b) production layer, (c) moderation layer, (d) reaction layer, (e) silicon detector, (f) alpha particle collimation. Layer thicknesses are not to scale.

of the target. To date, only experiments using triple mixtures of isotopes have produced results which test the theoretical calculations of the strongest resonances, but the analysis relies very much upon a complete kinetic simulation of muonic interactions in the target to model the time distributions of transient phenomena[14].

Solid targets with spatially separated layers provide another way to measure the rate and energy dependence of resonant muon molecular formation, using the time-of-flight (TOF) technique. By careful design of the target, the kinetic energy range of most interest becomes accessible, using the energy gained in transfer of the muon to the heavier isotope and the phenomenon of, in certain cases, a very low rate of energy loss (the Ramsauer-Townsend mechanism). Comparatively little tritium is required, simplifying some technical aspects of target design. The TOF method provides results with different systematic limitations than thermal or triple mixture target experiments. In addition, direct detection of charged fusion products is quite straightforward; background considerations make this a very attractive experimental advantage. However, the analysis still relies upon accurate simulation of complex muonic interactions in the target structure, with the added requirement of geometrical tracking. The understanding of several related processes is still critical to successful analysis of the time-of-flight distributions.

In the measurements described here, muonic atoms are emitted from a production layer with an energy distribution equivalent to thermal energies of many thousands of degrees. Another layer, called a reaction layer, can be created with a different isotopic composition, where muonic interactions at elevated energies can be studied. The separation of the functions of production and reaction helps to separate the processes of interest, which for some measurements is a great experimental advantage. In addition, there is a moderation layer which can be used between the production and reaction layers to adjust. the energy of $\mu t$ to a range more appropriate for the reaction of interest. 


\section{Formation and emission of muonic atoms}

The target region (Fig. 2) was bounded by two parallel gold foils, each of nominal thickness $0.051 \mathrm{~mm}$, separated by a distance of $17.9 \mathrm{~mm}$. Each foil was mounted to cover the $65 \mathrm{~mm}$ diameter opening in a gold plated copper frame, which was kept at about $3 \mathrm{~K}$ by attachment to the cold stage of a cryostat. Hydrogen isotope mixtures could be deposited independently (with cross contamination of less than $10^{-3}$ ) on the adjacent surfaces of either of the foils, and layer thicknesses were calibrated from separate studies of alpha particle energy loss[15]. Further details of the target design and construction can be found elsewhere[16].

On the gold foil through which the muon beam entered, a relatively thick production layer was first deposited, consisting of $(3.5 \pm 0.2) \mathrm{mg} \mathrm{cm}^{-2}(0.4 \mathrm{~mm}$ assuming a solid atomic density relative to liquid hydrogen of $\phi=1.24)$ of protium with a concentration of tritium, $c_{t}$, of either 0.001 or 0.002 . The two values were used to enable another measurement to be performed simultaneously[3]. A beam of negative muons was adjusted in momentum to $24 \mathrm{MeV} / \mathrm{c}$ to maximize the number of muons stopping in the layer. The incident rate was approximately $5 \times 10^{3} \mathrm{~s}^{-1}$, with momentum spread of $4 \%$ full width at half maximum. The muon decay time distribution for an equivalent pure protium target, in which the stops in hydrogen can be clearly identified by the characteristic muon lifetime of $2.2 \mu \mathrm{s}$, showed that a fraction $0.30 \pm 0.01$ stopped in the production layer. Most of the muons initially formed $\mu p$, but transferred to form $\mu t$ with a kinetic energy of $45 \mathrm{eV}$ due to the difference in reduced mass (assuming ground state transfer). Elastic scattering calculations on molecules[17] show that the cross section is less than a few times $10^{-20} \mathrm{~cm}^{2}$ at this energy, and drops even further to a minimum near a laboratory energy of $10 \mathrm{eV}$, a manifestation of the Ramsauer-Townsend effect. The scattering is small and forwardpeaked, resulting in macroscopic displacements from the point of transfer and significant emission of $\mu t$ from the surfaces of the production layer. In general, the time scale of emission is determined largely by the mean transfer time, typically $0.1 \mu$ s depending on $c_{t}[3]$. The analogous phenomenon has been observed and reported for emission of $\mu d[18,19]$.

\section{The time-of-flight technique}

The use of flight time to determine the energy distribution for muon molecular formation is made possible by several aspects of the muonic processes and the experimental design. Simulations were extremely important to calculate various effects and to optimize the target composition and geometry. They are also required to interpret the data, because it is generally not possible to invert the time of flight to yield the energy in a unique way. A great amount of detail regarding the simulation can be found elsewhere $[8,9]$.

It is necessary that the flight time is closely related to the muonic atom's energy. The speed of $\mu t$ of energy $1 \mathrm{eV}$ is about $0.8 \times 10^{6} \mathrm{~cm} \mathrm{~s}^{-1}$, so the emission time scale is short compared with the flight time over $17 \mathrm{~mm}$ for a $\mu$ t atom up to several $\mathrm{eV}$. The resonant rates for molecular formation are calculated[11] to be $\sim 10^{9} \mathrm{~s}^{-1}$ at liquid hydrogen density, and subsequent fusion occurs at $\sim 10^{12} \mathrm{~s}^{-1}$, so if molecular formation occurs in a reaction layer on the second gold foil separated in space from the production layer, the time interval from detection of muon arrival to detection of fusion is dominated by the flight time. Therefore, one should be able to infer the energy of molecular formation from the flight time. The predicted resonances in the energy range of 0.3 to $0.7 \mathrm{eV}$ should 
appear at times greater than $3 \mu$ s in the fusion time distribution. The somewhat weaker resonances above $0.8 \mathrm{eV}$ should be seen between 2 and $3 \mu \mathrm{s}$, with greater efficiency because of reduced in-flight loss of $\mu t$ to muon decay.

In addition, it is crucial that the molecular formation rate at resonance is not small compared to rates of other processes, such as elastic scattering of $\mu t$ on $\mathrm{D}_{2}$, which compete and lead to a change of the muonic atom's energy. The indirect process, whereby the $\mu t$ loses energy in the reaction layer prior to molecular formation, presents an unavoidable complication to the interpretation of the time of flight; in this case, the time of flight is not an indication of the energy at which molecular formation takes place. A careful selection of the reaction layer thickness is therefore important. It must be a substantial fraction of a characteristic interaction length for molecular formation, but not so thick that scattering at energies away from the resonance dominate the TOF spectrum. We have chosen $0.022 \mathrm{mg} \mathrm{cm}^{-2}(0.001 \mathrm{~mm}$ assuming a relative atomic density for solid deuterium of $\phi=1.43$ ) as the reaction layer thickness for the TOF measurements.

The simulations showed that the distribution of energy for $\mu t$ emitted from the production layer is significantly higher than the predicted resonance energy, so a moderation layer consisting of $0.10 \mathrm{mg} \mathrm{cm}^{-2}(0.005 \mathrm{~mm}$ at $\phi=1.43)$ of $\mathrm{D}_{2}$ was frozen onto the surface of the production layer. This moderation layer, or overlayer as it might be called, slows the $\mu t$ emitted from the production layer. Although a significant proportion of $\mu t$ atoms also form $d \mu t$ in the moderation layer and lead to a strong signal from fusion, the time distribution is such that virtually all such events occur within $1 \mu$ s of the muon arrival time, well before the range of interest for TOF.

\section{Preliminary results}

In the data presented here, the $3.5 \mathrm{MeV}$ alpha particle from fusion in the reaction layer was detected with good time and energy resolution in one of two ion-implanted silicon detectors near the target region [Fig. 2]. The $14 \mathrm{MeV}$ neutron was also detected and can provide further fusion time information.

If one accepts alpha events from a wide time range with respect to the muon arrival time, a broad, asymmetric energy distribution is obtained as in Fig. 3. The calibration is such that one channel is very close to $1 \mathrm{keV}$, with no offset. The distributions from the two silicon detectors are nearly identical, confirming the symmetry of the apparatus and the muon beam distribution, and have been added together in the figure. The background obtained from a separate run with no $\mathrm{D}_{2}$ present has no such peak. Most events come from fusion in the moderation layer, and the width is due to energy loss of the alphas as they exit the layer at the angles required for detection in the silicon detectors. A small feature at $3 \mathrm{MeV}$ (channel 3000) in the energy distribution is due to protons from $d d$ fusion induced by muons stopping directly in either the moderation or reaction layer.

Very few of the displayed events occur beyond $1.5 \mu$ s after the arrival of the muon. To distinguish the TOF events in the comparatively thin reaction layer, the time range of 1.5 to $5.0 \mu \mathrm{s}$ is selected, and background data obtained without the reaction layer (but with the moderation layer) is subtracted, giving the alpha energy spectrum of Fig. 4. Again, the two detectors give nearly identical results, so they have been summed. The energy distribution is nearly symmetric and much narrower, because the alphas do not lose much energy in the layer.

By applying energy cuts as shown in Fig. 4, and subtracting background determined without the reaction layer, the time-of-flight distribution of Fig. 5 is obtained. The error 


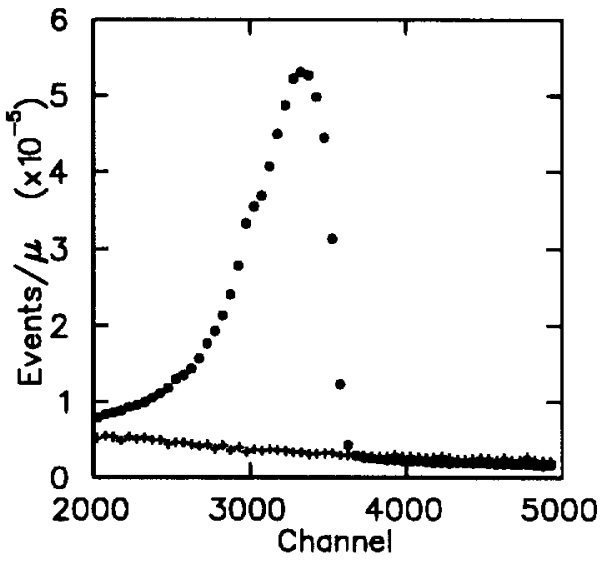

Figure 3: The energy deposited in the silicon detectors, normalized to the number of muons, for a time range including early times where fusion in the moderation layer dominates (filled circles). Background from a separate run with no moderation or reaction layers is also shown (crosses).

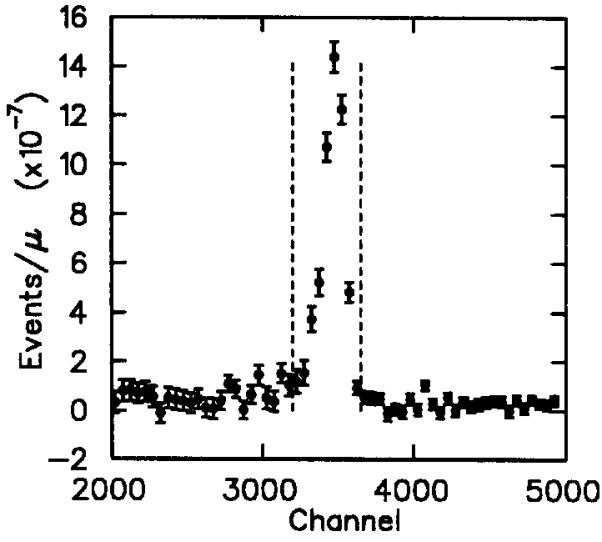

Figure 4: The energy deposited in the silicon detectors, normalized to the number of muons, for a time range of 1.5 to $5 \mu \mathrm{s}$, corresponding to times of flight to the target $\mathrm{D}_{2}$ layer. Background has been subtracted. The dashed lines show the energy range selected for TOF analysis.

bars include the contribution from subtraction of the background, and are statistical only. The data is compared to the results of the simulation with a number of assumptions. Since the number of events is significantly lower than indicated by experiment, the theoretical resonance structure of Fig. 1 has been arbitrarily scaled by 0.5 . The silicon detectors have been assigned an efficiency determined by a simulation of the solid angle, which is 0.028 for each device, for a total of 0.056 . The fraction of muons stopping in the production layer is taken from the measurements described previously to be 0.30 . Electronic and data acquisition dead times have also been included in the calculation of the number of muons corresponding to experiment.

If our prediction is correct for the processes of muon tranfer to tritium, $\mu t$ transport via the Ramsauer-Townsend mechanism in the production layer, $\mu t$ energy loss in $D_{2}$ by elastic scattering in both moderation and reaction layers, and of muon molecular resonant formation with the scaling of 0.5 applied, and if the experimental factors have been taken into account correctly, the simulation should match the data in intensity and shape. It clearly fails to do so in both aspects. With the arbitrary scaling factor, the shape and intensity are approximately correct except for a peak in the simulated distribution in the range of $3.0-4.5 \mu \mathrm{s}$ which is the time of flight corresponding to the strongest resonances near $0.5 \mathrm{eV}$.

Analysis is now underway to check the normalization procedures, and to determine which processes in the simulation, if any, might be adjusted to improve agreement. Data was taken with other target configurations which will help to distinguish which of the many processes are likely candidates. For example, Fig. 6 shows another comparison of simulated and experimental data for a considerably thicker reaction layer of $0.15 \mathrm{mg} \mathrm{cm}^{-2}$ $(0.007 \mathrm{~mm}$ at $\phi=1.43)$. Here, the resonance structure is expected to play a less important role due to the dominance of the indirect mechanism. In the approximation that the layer 


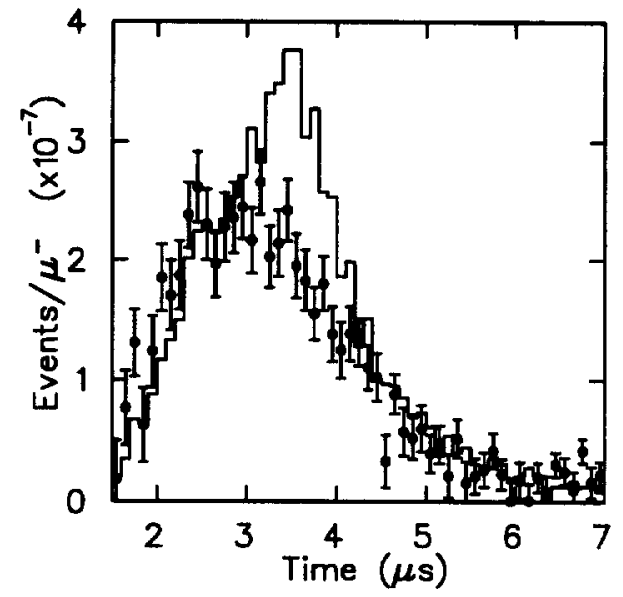

Figure 5: Experimental TOF data after subtraction of background (filled circles) compared to simulation results, with molecular formation scaled by 0.5 , for the time of flight distribution (histogram).

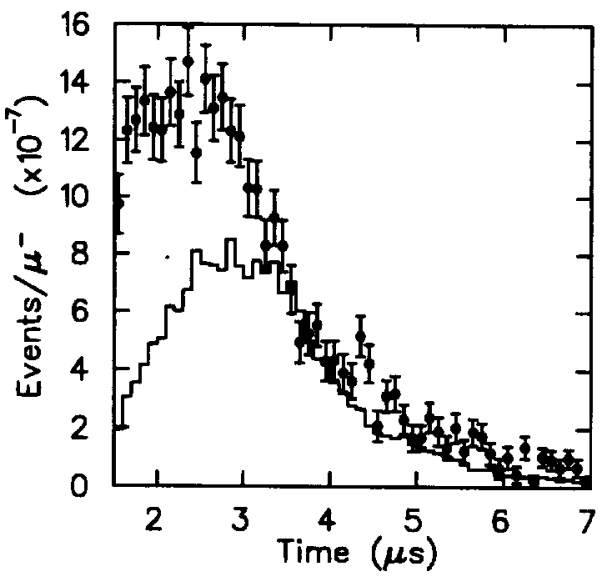

Figure 6: Comparison of data and simulation results as for the previous figure, but for a thicker reaction layer where the indirect process is expected to dominate.

is thick enough to stop all $\mu t$, and that molecular formation occurs quickly compared to the other contributions to the measured TOF, the distribution can be interpreted as the time of arrival of $\mu t$ at the reaction layer; the conclusion then is that there are more $\mu t$ with higher energy than the simulation predicts, pointing to deficiencies in the modelling of $\mu t$ energy loss in the production and moderation layers.

There are also other pieces of information which have yet to be included. The intensity of fusion events in the moderation layer, whose time structure was reported in [3], does not depend critically on the resonance structure and can be analyzed for consistency with the simulation. The imaging of $\mu t$ emitted from the production layer with no $D_{2}$ present is most sensitive to the emission energy distribution, and can also be analyzed for consistency (see $[18,19]$ for a description of imaging data for $\mu d$ emission). Finally, although not considered to be important because of the higher energies of the muonic atoms in the TOF experiment, solid state effects may play some role which is not explicitly included in the simulation.

\section{Acknowledgments}

The authors gratefully acknowledge the support of the Canadian Natural Sciences and Engineering Research Council (NSERC), the US National Science Foundation, the Swiss National Science Foundation, and a NATO Linkage Grant LG 930162.

\section{References}

[1] P. E. Knowles et al., Muon catalyzed fusion in deuterium at $3 \mathrm{~K}$, proceedings of this con- 
ference.

[2] V. G. Zinov, Measurement of spin and temperature dependence of $d d \mu$ molecule formation rate in gaseous, liquid, and solid deuterium, proceedings of this conference.

[3] F. Mulhauser et al., Measurement of the muon transfer rate $\lambda_{p t}$ and molecular formation $\lambda_{p p \mu}$ in solid hydrogen targets, proceedings of this conference.

[4] R. Jacot-Guillarmod et al., Muon molecular formation and transfer rate in solid hydrogendeuterium mixtures, proceedings of this conference.

[5] P. Strasser et al., Muon catalyzed fusion experiments in thin deuterium films and progress towards slow negative muon production, proceedings of this conference.

[6] M. C. Fujiwara et al., Measuring sticking and stripping in muon catalyzed $d t$ fusion with multilayer solid films, proceedings of this conference.

[7] R. Jacot-Guillarmod et al., Investigation of muonic hydrogen isotopes scattering (TRIUMF Expt. 742), proceedings of this conference.

[8] V. E. Markushin, Muonic atom emission into vacuum from inhomogeneous mixtures of hydrogen isotopes and kinetics of muon catalyzed fusion in multilayered target systems, preprint PSI-PR-94-38, PSI, Villigen, 1994.

[9] V. E. Markushin, Muonic atoms and muon catalyzed fusion in inhomogeneous mixtures of hydrogen istotopes, proceedings of this conference.

[10] J. Woz̀niak et al., Study of muonic hydrogen transport in TRIUMF Expt. 742 by Monte Carlo method, proceedings of this conference.

[11] M. P. Faifman and L. I. Ponomarev, Resonant formation of $d t \mu$ mesic molecules in the triple $\mathrm{H}_{2}+\mathrm{D}_{2}+\mathrm{T}_{2}$ mixture, Phys. Lett. B, 265, (1991) 201-206.

[12] M. P. Faifman et al., Quadrupole corrections to matrix elements of transitions in resonant reactions of mesic molecule formation, proceedings of this conference.

[13] Yu. V. Petrov et al., Mesomolecule formation in $t \mu+H D\left(D_{2}\right)$ reactions, Phys. Lett. B, 34 (1994) 266-270.

[14] M. Jeitler et al., Epithermal effects in muon-catalyzed $d t$ fusion: Comparison of experimental data with theoretical calculations, Phys. Rev. A, 51 (1995) 2881-2898.

[15] M. C. Fujiwara et al., Characterization of solid hydrogen target via alpha particle energy loss, proceedings of this conference. See also M. C. Fujiwara, M.A.Sc. Thesis, University of British Columbia, 1994.

[16] P. E. Knowles et al., Producing $\mu^{-} \mathrm{d}$ and $\mu^{-} \mathrm{t}$ in vacuum, Hyp. Int., 82 (1993) 521-527; P. E. Knowles et al., A windowless frozen hydrogen target system, preprint TRI-PP-95-33, TRIUMF, Vancouver, 1995 (accepted for publication in Nucl. Instr. and Meth.).

[17] A. Adamczak et al., Atlas of cross sections for muonic hydrogen scattering on hydrogen isotope molecules, report WMM 94-1, College of William and Mary, Williamsburg, 1994.

[18] B. M. Forster et al., Production of slow muonic hydrogen isotopes in vacuum, Hyp. Int., 65 (1990) 1007-1014.

[19] G. M. Marshall et al., Emission of muonic hydrogen isotopes from solid hydrogen layers into vacuum, in Proceedings of an International Symposium on Muon Catalyzed Fusion $\mu$ CF-89, J. D. Davies ed., report RAL-90-022, Rutherford Appleton Laboratory, 1990. 